EPV080/\#499 EVALUATION OF THE PELVIC AUTONOMIC NERVE FUNCTION AND QUALITY OF LIFE AFTER TYPE C1 HYSTERECTOMY USING INTRAOPERATIVE NERVE MONITORING FOR EARLY-STAGE CERVICAL CANCER

${ }^{1} \mathrm{SJ}$ Park*, ${ }^{2} \mathrm{~J} \mathrm{Kim},{ }^{3} \mathrm{~S}$ Lee, ${ }^{2} \mathrm{HS}$ Kim, ${ }^{1} \mathrm{~J}-\mathrm{W}$ Kim. ${ }^{1}$ Seoul National University College of Medicine, Obstetrics and Gynecology, Seoul, Korea, Republic of; ${ }^{2}$ Seoul National University Hospital, Department of Obstetrics and Gynecology, Seoul, Korea, Republic of; ${ }^{3}$ Keimyung University School of Medicine, Department of Obstetrics and Gynecology, Seoul, Korea, Republic of

\subsection{6/ijgc-2021-IGCS.148}

Objectives We evaluated the pelvic autonomic nerve function and quality of life after type C1 hysterectomy using intraoperative nerve monitoring (INM) for early-stage cervical cancer.

Methods From 2015 to 2016, 11 patients with stage IB cervical cancer were enrolled prospectively to receive type $\mathrm{C} 1$ hysterectomy using INM (NCT02952183). After confirming that the hypogastric, pelvic splanchnic, and vesical branches of the pelvic plexus, we evaluated their function by measuring the bladder, vagina, and rectum pressure electrical stimulation of the pelvic autonomic nerves. Moreover, we investigated the quality of life related to the pelvic organ function before and three months after surgery.

Results Bilateral pelvic autonomic nerves were preserved in all patients. When we stimulated parasympathetic nerves, we found that periodic and regular contraction of the bladder and rectum. In contrast, the stimulation of sympathetic nerves decreased the interval to contraction and the duration of contraction and increased the maximal pressure and frequency of contraction despite no consistent change of the vaginal pressure. Moreover, despite the normal residual urine volume, the sustained voiding difficulty, abdominal distension, discomfort, and fecal incontinence increased after surgery, whereas there was no consistent change in the sexual function.

Conclusions The pelvic sympathetic and parasympathetic nerves may show the opposite effect on each other for the bladder and rectal function. Furthermore, the quality of life related to the bladder and rectum may decrease despite the pelvic autonomic nerve preservation using INM during type C1 hysterectomy for stage IB cervical cancer.

\section{EPV081/\#509 SENTINEL LYMPH NODE MAPPING USING INTRA- ABDOMINAL INDOCYANINE GREEN INJECTION IN OPEN SURGERY FOR CERVICAL CANCER: A NOVEL TECHNIQUE OF INDOCYANINE GREEN INJECTION BY ABDOMINAL APPROACH}

${ }^{1}$ YM Kim*, ${ }^{1}$ HH Choi, ${ }^{1}$ CK Lim, ${ }^{1}$ S-W Lee, ${ }^{1}$ JY Park, ${ }^{1}$ DY Kim, ${ }^{1} J H$ Kim, ${ }^{2}$ K-R Kim, ${ }^{1} Y T$ Kim. ${ }^{1}$ Asan Medical Center, University of Ulsan, Department of Obstetrics and Gynecology, Seoul, Korea, Republic of; ${ }^{2}$ Asan Medical Center, University of Ulsan, Department of Pathology, Seoul, Korea, Republic of

10.1136/ijgc-2021-IGCS. 149

Objectives Recently, open radical hysterectomy in early-stage cervical cancer has been preferred after the LACC trial was published. and the role of sentinel lymph node (SLN) is increasing in the surgical treatment of cervical cancer. We evaluated the feasibility the of SLN mapping by intra- abdominal Indocyanine green (ICG) injection during open surgery for cervical cancer.

Methods We performed retrospective study at a single center. The novel technique is done by using ICG which was injected in bilateral side between the isthmus and cervix before or after bladder peritoneum dissection. SLN in open surgery was detected with SPY Portable Handheld Imager (SPY-PHI) camber (Stryker, Kalamazoo, Michigan, US). All patients underwent open SLN mapping followed by radical surgery (hysterectomy or trachelectomy) and systemic pelvic lymphadenectomy

Results From June 2020 to April 2021, thirty-three patients, newly diagnosed FIGO 2018 stage IA1 to IIIC1p cervical cancer who underwent open surgeries, were included in this study. of these patients, 29 (87.9\%) radical hysterectomy and $4(12.1 \%)$ underwent radical trachelectomy. Twenty three (69.7\%) patients showed bilateral SLN detection, and the proportion of patient with at least unilateral SLN detection was $97 \%$ (32/33). Compared to the final pathology results were all consistent with the frozen biopsy results of SLN mapping. Per-patient sensitivity (5/5) and negative predictive value (28/ 28) of SLN biopsy were both $100 \%$.

Conclusions SLN mapping with ICG in open method can be considered as a feasible, reliable technique to be used in open surgery for cervical cancer with high detection rate.

\section{EPV082/\#511 ROUTINE PELVIC LYMPH NODE FROZEN SECTION EXAMINATION IN PREVENTING INEFFECTIVE DUAL MODALITY MANAGEMENT IN EARLY-STAGE CERVICAL CANCER}

${ }^{1} \mathrm{C}$ Pappa*, ${ }^{2} \mathrm{~S}$ Smyth, ${ }^{1} \mathrm{H}$ Jiang, ${ }^{3} \mathrm{C}$ Johnson, ${ }^{4} \mathrm{M}$ Mccole, ${ }^{4} \mathrm{~S}$ Damato, ${ }^{1} \mathrm{~S}$ Wood, ${ }^{1} \mathrm{H}$ Soleymani Majd, ${ }^{1} \mathrm{M}$ Alazzam. ${ }^{1}$ Oxford University Hospital NHS Foundation Trust, Gynaecology Oncology Department, Oxford, UK; ${ }^{2}$ Oxford University Hospitals NHS Foundation Trust, Gynaecological Oncology, Oxford, UK; ${ }^{3}$ Oxford University Hospitals NHS Foundation Trust, Radiology, Oxford, UK; ${ }^{4}$ Oxford University Hospitals NHS Foundation Trust, Oxford, UK, Cellular Pathology, Oxford, UK

\subsection{6/ijgc-2021-IGCS. 150}

Objectives Cervical cancer is one of the most common malignancies mainly affecting young women. Surgical management including pelvic lymphadenectomy comprise the cornerstone in the treatment of early-stage cervical carcinomas. Our aim is to evaluate the role of intraoperative pelvic lymph nodes FSE in the surgical management of early-stage cervical cancer to prevent ineffectual radical surgery and its potential complications.

Methods A retrospective study of 30 consquetive women aged between 23 and 82 years (mean age $37 \pm 7$ years) was conducted in our department. All women had a diagnosis of stage Ib1 or less cervical malignancy with a tumor size less than $20 \mathrm{~mm}$. Trachelectomy was performed when lymph nodes FSE in both pelvic sides were negative. In case of positive nodes in FSE any further surgical procedure was abandoned.

Results The mean number of pelvic PSE LNs excised bilaterally was $19 \pm 6$. Four out of 30 women (13.3\%) were found to have positive LNs at frozen section examination. No false positive cases were proved after the final paraffin histopathology examination. The mean time from LNs excision to FSE report was $82.3 \pm 20.4$ minutes. 